\title{
Nebivolol versus Carvedilol or Metoprolol in Patients Presenting with Acute Myocardial Infarction Complicated by Left Ventricular Dysfunction
}

\author{
Mehmet Ozaydin ${ }^{a}$ Habil Yucel ${ }^{b}$ Sule Kocyigit ${ }^{c}$ Mehmet Koray Adali ${ }^{d}$ \\ Fatih Aksoy ${ }^{\mathrm{e}}$ Fatih Kahraman $^{\mathrm{a}}$ Bayram Ali Uysal ${ }^{\mathrm{f}}$ Dogan Erdogan $^{\mathrm{a}}$ \\ Ercan Varola Abdullah Dogang \\ a Department of Cardiology, Suleyman Demirel University, Isparta, b State Hospital, Manisa, ' Servergazi State

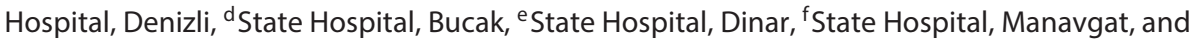 \\ ${ }^{9}$ Katip Celebi University, Izmir, Turkey
}

\section{Key Words}

Beta-blockers · Myocardial infarction · Major cardiac events

\section{Abstract}

Objective: The aim of this study was to evaluate the efficacy of nebivolol, carvedilol or metoprolol succinate on the outcome of patients presenting with acute myocardial infarction (AMI) complicated by left ventricular dysfunction. Subjects and Methods: Patients ( $n=172$, aged $28-87$ years) with $\mathrm{AMI}$ and left ventricular ejection fraction $\leq 0.45$ were randomized to the nebivolol $(n=55)$, carvedilol $(n=60)$ and metoprolol succinate $(n=57)$ groups. Baseline demographic and clinical characteristics and composite event rates of nonfatal Ml, cardiovascular mortality, hospitalization due to unstable angina pectoris or heart failure, stroke or revascularization during the 12-month follow-up were compared among the groups using the $x^{2}$ test, $t$ test or log-rank test as appropriate. Results: Baseline demographic and clinical characteristics were similar in the three groups. The composite end point during follow-up was lower in the patients treated with nebivolol than those treated with metoprolol
(14.5 vs. $31.5 \% ; p=0.03$ ). However, event rates were similar between the patients treated with carvedilol and those treated with the metoprolol ( 20.3 vs. $31.5 \%, p>0.05)$ and between the patients treated with nebivolol and carvedilol (14.5 vs. $20.3 \%, p>0.05)$. Conclusion: The patients treated with nebivolol experienced 12-month cardiovascular events at a lower rate than those treated with metoprolol succinate. However, event rates were similar between the carvedilol and the metoprolol succinate groups and between the nebivolol and the carvedilol groups.

(c) 2016 S. Karger AG, Basel

\section{Introduction}

Beta-blockers (BBs) decrease mortality and morbidity and improve left ventricular (LV) function in chronic heart failure [1-3]. They also decrease ischemic events and mortality after acute myocardial infarction (AMI) with greater beneficial effects in those who develop LV dysfunction and/or heart failure $[1,4]$. Four BBs have been shown to be effective in heart failure: metoprolol,

\begin{tabular}{ll}
\hline KARGER & $\begin{array}{l}\text { ○ 2016 S. Karger AG, Basel } \\
1011-7571 / 16 / 0254-0316 \$ 39.50 / 0 \quad \text { Karger }\end{array}$ \\
E-Mail karger@karger.com & $\begin{array}{l}\text { Thisis an Open Access article licensed under the terms of the } \\
\text { Creative Commons Attribution-NonCommercial 3.0 Un- } \\
\text { ported license (CC BY-NC) (www.karger.com/OA-license), } \\
\text { applicable to the online version of the article only. Distribu- } \\
\text { tion permitted for non-commercial purposes only. }\end{array}$
\end{tabular}

Dr. Mehmet Ozaydin

Suleyman Demirel University, Tip Fakultesi Hastanesi

Kardiyoloji AD, Cunur

TR-32200 Isparta (Turkey)

E-Mail mehmetozaydin@ hotmail.com 
Fig. 1. Flow diagram of the inclusion of patients in the study.

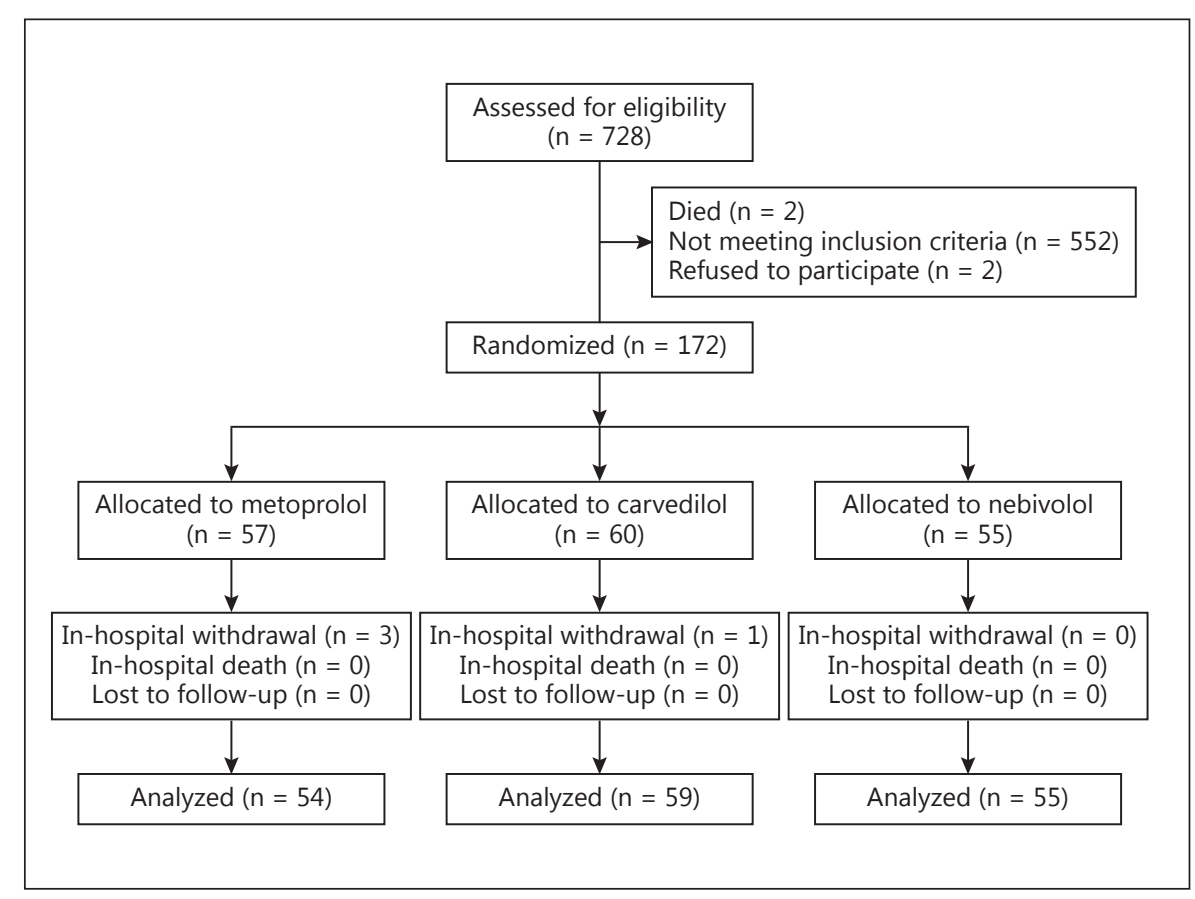

carvedilol, bisoprolol and nebivolol. Metoprolol, bisoprolol and nebivolol are $\beta_{1}$-selective BBs. However, carvedilol has $\beta_{1}$ and $\beta_{2}$ receptor and $\alpha_{1}$ receptor blocker and $\mathrm{M}_{2}$ receptor upregulation properties. Nebivolol has nitric oxide-releasing and vasodilatory properties and inhibits endothelial proliferation [5-7].

A comparison of different BBs in patients with LV dysfunction and/or heart failure showed divergent results. Carvedilol showed better results on clinical end points as compared with metoprolol in patients with chronic heart failure [2], but they showed similar effects on the outcomes in patients with AMI complicated by LV systolic dysfunction [7]. A comparison of metoprolol and nebivolol showed that metoprolol deteriorated LV systolic function in patients with systolic dysfunction but not nebivolol [8]. However, carvedilol and nebivolol showed similar efficacy, including clinical end points in patients with systolic dysfunction and hypertension [9] or chronic heart failure [10]. In a meta-analysis [11], carvedilol was shown to reduce all-cause mortality as compared with $\beta_{1}$-selective BBs including metoprolol and nebivolol in randomized direct comparison trials in patients with heart failure. No headto-head comparison of nebivolol, carvedilol and metoprolol has been conducted in ischemic LV dysfunction. Therefore, the aim of this study was to compare nebivolol with carvedilol or metoprolol succinate in patients presenting with AMI and an LV ejection fraction $\leq 0.45$.

Myocardial Infarction and Major Cardiac Events

\section{Subjects and Methods}

This was a single-center, randomized, active-controlled and end point-blinded study. Patients with a diagnosis of AMI based on clinical, electrocardiographic and cardiac biomarker criteria [12] and an echocardiographic LV ejection fraction $\leq 0.45$ were included. Transthoracic echocardiography was performed in each patient before the randomization, and the echocardiographic LV ejection fraction was determined by the Simpson method [13]. A total of 728 patients presenting with ST elevation or non-ST elevation MI between March 2009 and March 2012 were screened. The inclusion criteria were patients $>18$ years old presenting with AMI and an ejection fraction $\leq 0.45$. Exclusion criteria were patients with an ejection fraction $>0.45$, Killip class IV heart failure, unstable angina pectoris, prior MI, previous $\mathrm{BB}$ or a-blocker use, baseline heart rate $<60 \mathrm{bpm}$, second- or third-degree atrioventricular block, systolic blood pressure $<90 \mathrm{~mm} \mathrm{Hg}$, severe chronic obstructive pulmonary disease and symptomatic peripheral arterial disease. Based on these criteria, 552 patients were excluded: 352 due to ejection fraction $>0.45,133$ because of previous use of BBs, 22 with a heart rate $<60,12$ with systolic blood pressure $<90 \mathrm{~mm}$ $\mathrm{Hg}, 4$ with second- or third-degree atrioventricular block, 6 with Killip class IV heart failure, 15 due to unstable angina pectoris, 2 with severe chronic obstructive pulmonary disease, 4 because of prior heart failure and 2 with previous MI. In addition, 2 patients died before randomization and 2 refused to participate. Therefore, 172 patients (aged 28-87 years) were included in this study (fig. 1; table 1). Of the eligible patients, 57 were randomized to metoprolol succinate, 60 to carvedilol and 55 to nebivolol. Selection for these groups was conducted using numbers in sealed envelopes. Three patients in the metoprolol succinate group (bronchospasm, $\mathrm{n}=1$; atrioventricular block, $\mathrm{n}=2$ ) and 1 patient in the carvedilol 
Table 1. Baseline characteristics

\begin{tabular}{|c|c|c|c|c|}
\hline Characteristics & $\begin{array}{l}\text { Metoprolol succinate } \\
(\mathrm{n}=57)\end{array}$ & $\begin{array}{l}\text { Carvedilol } \\
(\mathrm{n}=60)\end{array}$ & $\begin{array}{l}\text { Nebivolol } \\
(\mathrm{n}=55)\end{array}$ & $\mathrm{p}$ \\
\hline Age, years & $59 \pm 13$ & $59 \pm 12$ & $61 \pm 11$ & 0.98 \\
\hline Male gender & $48(84.2)$ & $47(78.3)$ & $50(90.9)$ & 0.18 \\
\hline Smoking & $33(57.9)$ & $38(63.3)$ & $28(50.9)$ & 0.29 \\
\hline Hypertension & $21(36.8)$ & $16(26.7)$ & $17(30.9)$ & 0.48 \\
\hline ST elevation MI & $55(96.5)$ & $58(96.7)$ & $52(90.9)$ & 0.72 \\
\hline Anterior & $44(77.2)$ & $47(78.3)$ & $36(65.4)$ & 0.50 \\
\hline Nonanterior & $11(19.3)$ & $11(18.3)$ & $14(25.5)$ & 0.30 \\
\hline \multicolumn{5}{|l|}{ Killip class } \\
\hline Class I & $43(75.4)$ & $44(73.3)$ & $41(74.5)$ & 0.80 \\
\hline Class II & $11(19.3)$ & $12(20)$ & $10(18.2)$ & \\
\hline HDL cholesterol, mg/dl & $41 \pm 9$ & $41 \pm 6$ & $41 \pm 9$ & 0.97 \\
\hline LDL cholesterol, mg/dl & $108 \pm 42$ & $95 \pm 27$ & $111 \pm 24$ & 0.37 \\
\hline Ejection fraction, $\%$ & $35(20-45)$ & $35(25-45)$ & $35(30-45)$ & 0.54 \\
\hline Baseline systolic BP, mm Hg & $130(90-150)$ & $120(80-200)$ & $135(90-180)$ & 0.26 \\
\hline Baseline diastolic BP, $\mathrm{mm} \mathrm{Hg}$ & $80(60-100)$ & $75(50-120)$ & $80(55-100)$ & 0.28 \\
\hline Baseline heart rate, beats $/ \mathrm{min}$ & $75(61-110)$ & $80(60-120)$ & $76(60-120)$ & 0.43 \\
\hline Peak CK-MB & $100(41-520)$ & $111(26-573)$ & $143(45-380)$ & 0.30 \\
\hline \multicolumn{5}{|l|}{ Medications before hospitalization } \\
\hline ACEI and/or ARB & $8(14)$ & $7(11.7)$ & $7(12.7)$ & 0.92 \\
\hline Acetyl salicylic acid & $4(7)$ & $6(10)$ & $4(7.3)$ & 0.84 \\
\hline Statins & $6(10.5)$ & $7(11.7)$ & $5(9.1)$ & 0.95 \\
\hline Oral antidiabetics & $5(8.8)$ & $5(8.3)$ & $2(3.6)$ & 0.46 \\
\hline Clopidogrel & $54(93.1)$ & $61(100)$ & $54(98.2)$ & 0.90 \\
\hline Recanalization in ST segment elevation MI & & & & 0.48 \\
\hline Thrombolytic & $22(40.1)$ & $23(39.7)$ & $21(38.1)$ & \\
\hline Streptokinase & $8(14.5)$ & $10(17.2)$ & $9(16.4)$ & \\
\hline $\mathrm{t}-\mathrm{PA}$ & $14(25.6)$ & $13(22.5)$ & $13(23.6)$ & \\
\hline Primary PCI & $21(38.1)$ & $21(36.2)$ & $19(34.5)$ & \\
\hline No recanalization & $12(21.8)$ & $14(24.1)$ & $11(20)$ & 0.68 \\
\hline Rescue PCI & $7(12.2)$ & $8(13.3)$ & $6(10.9)$ & 0.74 \\
\hline Treatment during index event & & & & 0.76 \\
\hline Medical treatment & $14(24.2)$ & $15(24.6)$ & $13(23.6)$ & \\
\hline PCI & $31(53.4)$ & $35(57.4)$ & $35(63.6)$ & \\
\hline CABG & $13(22.4)$ & $11(18)$ & $7(12.7)$ & \\
\hline $\begin{array}{l}\text { Implantable cardioverter defibrillator implantation } \\
\text { (for primary prevention) }\end{array}$ & $2(3.5)$ & $3(5.0)$ & $2(3.6)$ & 0.90 \\
\hline
\end{tabular}

Data are presented as the mean $\pm \mathrm{SD}$, median (range) or $\mathrm{n}(\%)$.

$\mathrm{HDL}=$ High-density lipoprotein; $\mathrm{LDL}=$ low-density lipoprotein; $\mathrm{CK}-\mathrm{MB}=$ creatine kinase myocardial band; $\mathrm{BP}=$ blood pressure; $\mathrm{ACEI}=$ angiotensin-converting enzyme inhibitor; $\mathrm{ARB}=$ angiotensin receptor blocker: $\mathrm{t}-\mathrm{PA}=$ tissue plasminogen activators; $\mathrm{CABG}=$ coronary artery bypass surgery. 
Table 2. Follow-up findings

\begin{tabular}{|c|c|c|c|c|c|c|c|}
\hline Characteristics & $\begin{array}{l}\text { Metoprolol } \\
\text { succinate }\end{array}$ & Carvedilol & Nebivolol & $\begin{array}{l}\mathrm{p} 1 \\
\text { overall }\end{array}$ & $\begin{array}{l}\text { p2 } \\
\text { neb vs. met }\end{array}$ & $\begin{array}{l}\text { p3 } \\
\text { neb vs. car }\end{array}$ & $\begin{array}{l}\mathrm{p} 4 \\
\text { car vs. met }\end{array}$ \\
\hline \multicolumn{8}{|l|}{ Tolerability } \\
\hline $\mathrm{n}$ & 57 & 60 & 55 & & & & \\
\hline BB withdrawal & $3(5.3)$ & $1(1.7)$ & 0 & & & & \\
\hline \multicolumn{8}{|l|}{ The reason for withdrawal } \\
\hline \multicolumn{8}{|l|}{ 12-month follow-up } \\
\hline $\mathrm{n}$ & 54 & 59 & 55 & & & & \\
\hline Primary composite end point ${ }^{a}$ & $17(31.5)$ & $12(20.3)$ & $8(14.5)$ & 0.03 & 0.03 & 0.46 & 0.20 \\
\hline Mortality & $1(1.9)$ & $1(1.7)$ & $2(3.6)$ & 0.78 & & & \\
\hline Nonfatal MI & $2(3.7)$ & $3(5.1)$ & $1(1.8)$ & 0.60 & & & \\
\hline
\end{tabular}

met $=$ Metoprolol; car = carvedilol; neb = nebivolol.

${ }^{a}$ Cardiovascular mortality, hospitalization due to unstable angina pectoris or heart failure, revascularization, nonfatal MI or stroke.

${ }^{\mathrm{b}}$ Hospitalization due to unstable angina pectoris or heart failure.

group (atrioventricular block, $\mathrm{n}=1$ ) were withdrawn from therapy (table 2). Therefore, 12-month follow-up was available for $168 \mathrm{pa-}$ tients (nebivolol, $\mathrm{n}=55$; carvedilol, $\mathrm{n}=59$; metoprolol succinate, $\mathrm{n}=54)$.

The BBs were started within the first 3 days after the onset of symptoms. Metoprolol, carvedilol and nebivolol were started at doses of $25 \mathrm{mg}$ once daily, $3.125 \mathrm{mg}$ twice daily and $1.25 \mathrm{mg}$ once daily, respectively. The dose was titrated up to a tolerable dose. In cases where the lowest dose was not tolerated, the study drug was stopped. Blood pressure and heart rate were checked every day and, depending on the patient's hemodynamic status, the metoprolol dose was increased gradually to 50, 100 and $200 \mathrm{mg}$ once daily, carvedilol was increased to $6.25,12.5$ and $25 \mathrm{mg}$ twice daily, and nebivolol was increased to $2.5,5$ and $10 \mathrm{mg}$ once daily. It was intended that the target dose would be reached within 1 month (200 mg once daily, $25 \mathrm{mg}$ twice daily and $10 \mathrm{mg}$ once daily for metoprolol, carvedilol and nebivolol, respectively). The patients were treated according to the most recent guidelines [14, 15]. Recanalization with either primary percutaneous coronary intervention (PCI) or thrombolytic treatment was performed in patients with ST elevation MI presenting within $12 \mathrm{~h}$ from the onset of the symptoms. Patients were taken to rescue PCI if no recanalization occurred at $1 \mathrm{~h}$ of thrombolytic treatment. If there was no contraindication, all patients received angiotensin-converting enzyme inhibitor or angiotensin receptor blocker, acetyl salicylic acid, clopidogrel, statins and low-molecular-weight heparin. All the patients without contraindications underwent coronary angiography (Schimatzu, Kyoto, Japan) by a femoral arterial approach within $24 \mathrm{~h}$ of the presentation. An implantable cardioverter defibrillator was implanted for primary prevention in patients with an ejection fraction $<30 \%$ and NYHA functional class II or III symptoms occurring $\geq 40$ days post-MI. The indication for cardiac re- synchronization therapy was heart failure and QRS duration $>120$ $\mathrm{ms}$ with a left bundle branch block pattern. However, as this criterion was not met, this therapy was not required in any patient. The study complies with the Declaration of Helsinki of 1975, as revised in 1983. The Institutional Review Board approved the study and written informed consent was obtained from all of the subjects. The primary end point was the composite of nonfatal MI, cardiovascular mortality, hospitalization due to unstable angina pectoris or heart failure, stroke and revascularization during the 12 months of follow-up. Patients were seen every week for the first month and at 3-month intervals thereafter for the assessment of end points and tolerability of the study drugs.

\section{Statistical Analysis}

The Kolmogorov-Smirnov test was used for the distribution of numeric variables and factors with a normal distribution are expressed as the mean \pm SD and were compared with analysis of variance (ANOVA), which was used to compare age, creatinine levels and lipid parameters. Variables without a normal distribution were expressed as the median (range) and were compared using the Kruskal-Wallis test for systolic and diastolic blood pressures, heart rate, peak creatine kinase myocardial band, and ejection fraction. Categorical variables are expressed as numbers and percentages. Continuous variables were compared using one-way ANOVA or the Kruskal-Wallis test, and categorical variables were compared using $\chi^{2}$ or Fisher's exact test as appropriate. Post hoc analyses were performed using the Scheffé correction test for controlling type 1 error if the overall test was significant. Post hoc analyses were not performed if the overall test did not reach a significant difference. The Kaplan-Meier method with the log-rank test was used to compare the probability of freedom from combined composite end points. Composite end point event rates were 


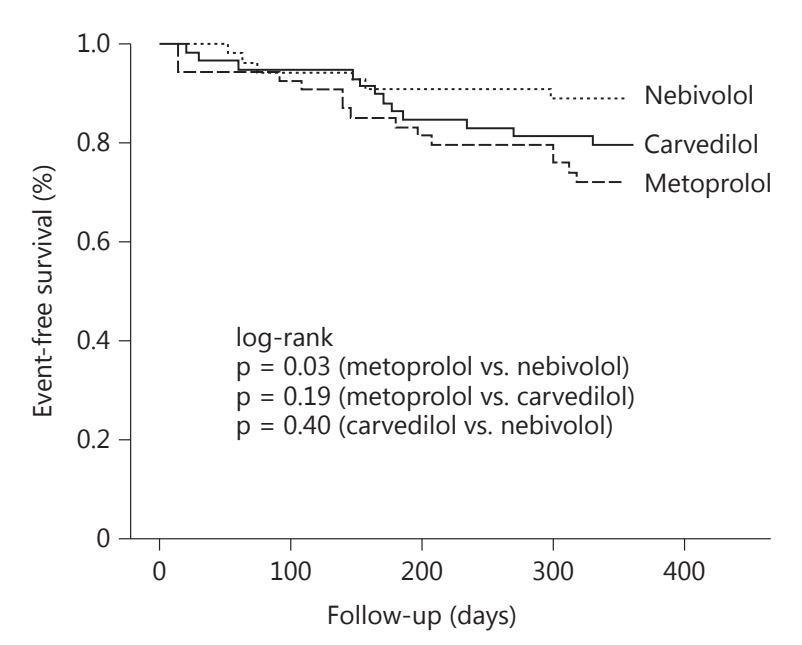

Fig. 2. Freedom from primary composite end points during the 12-month follow-up period.

assumed to be $30 \%$ in the metoprolol group, $15 \%$ in the carvedilol group and 7\% in the nebivolol group [7-10, 16-19], and were compared among the metoprolol, carvedilol and nebivolol groups. Given the fact that patients with coronary artery disease presenting with AMI were included in this study, we hypothesized that nebivolol would have the lowest event rates because it has nitric oxide-releasing and antiatherosclerotic properties. With this hypothesis, 47 patients in each group were required to detect significance with an alpha level of 0.05 , and a power of 0.80 . Analysis was performed on the intention-to-treat rule. A p value $<0.05$ was considered significant. SPSS 15 (SPSS Inc., Chicago, Ill., USA) was used for the analysis.

\section{Results}

Baseline findings were similar among the groups (all $\mathrm{p}$ values $>0.05$; table 1 ). The composite end point of nonfatal MI, cardiovascular mortality, hospitalization due to unstable angina pectoris or heart failure, stroke or revascularization during the 12-month follow-up period was lower in the nebivolol group $(\mathrm{n}=8,14.5 \%)$ than the metoprolol succinate group $(\mathrm{n}=17,31.5 \% ; \mathrm{p}=0.03)$. However, event rates were similar between the carvedilol and the metoprolol succinate groups and between the nebivolol and the carvedilol groups $(\mathrm{n}=12,20.3 \%$, vs. $\mathrm{n}=17$, $31.5 \% ; \mathrm{n}=8,14.5 \%$, vs. $\mathrm{n}=12,20.3 \%$, respectively; both $\mathrm{p}>0.05)$. The Kaplan-Meier survival analysis showed that freedom from composite end points was higher in the nebivolol group than in the metoprolol succinate group (log-rank $\mathrm{p}=0.03$ ), but was similar between the carvedilol and metoprolol succinate groups, and between the nebivolol and the carvedilol groups during the 12-month follow-up period (both log-rank $\mathrm{p}>0.05$; fig. 2). The mean daily doses reached for metoprolol, carvedilol and nebivolol were $57 \pm 27 \mathrm{mg}$ once a day, $10 \pm 3 \mathrm{mg}$ twice a day and $5.5 \pm 2 \mathrm{mg}$ once a day, respectively.

\section{Discussion}

In this study, patients with AMI and LV ejection fraction $\leq 45 \%$ treated with nebivolol experienced 12 -month cardiovascular events at a lower rate than those treated with metoprolol. However, event rates were similar between the carvedilol and the metoprolol groups and between the nebivolol and the carvedilol groups. It was shown in a previous study [8] that metoprolol but not nebivolol decreased cardiac output and increased systemic vascular resistance and pulmonary capillary wedge pressure in patients with systolic dysfunction. Similarly, nebivolol but not metoprolol inhibited cardiac NADPH oxidase activation and improved LV dysfunction, and nebivolol had a significantly more pronounced inhibitory effect than metoprolol on cardiomyocyte hypertrophy after MI [20]. Nebivolol was also found to be superior to atenolol in improving diastolic functions and the maximal exercise duration of patients with ischemic LV dysfunction [21]. However, in patients with nonischemic heart failure, both nebivolol and carvedilol improved LV diastolic functions and also performed similarly on follow-up [22]. On the other hand, a recent study showed that lung diffusion and exercise performance were higher with nebivolol than carvedilol, but carvedilol allowed better ventilation efficiency than nebivolol during exercise [23]. Although previous studies indicated the superiority of nebivolol over metoprolol, the present study is the first that shows lower long-term event rates with nebivolol than metoprolol in patients with AMI complicated by LV dysfunction. However, nebivolol and carvedilol, which had not been compared in this study population previously, had similar event rates in the present study. Nebivolol plays an important role in patients with reduced endothelial dysfunction, especially for those who have AMI, as it has nitric oxide-induced vasodilatory properties and may offer antiatherosclerotic activity by its inhibitory effects on oxidative stress and vascular smooth muscle proliferation [24]. By its vasodilatory effects, nebivolol decreases peripheral vascular resistance and increases stroke volume, which is very beneficial in heart failure [5]. 
Carvedilol has been found to have better results than other BBs in animal models of MI [25] and in decreasing cardiovascular event rates and increasing ejection fraction in patients with heart failure [26]. Similarly, a recent meta-analysis [11] indicated that carvedilol reduced allcause mortality as compared with atenolol, bisoprolol, metoprolol and nebivolol in randomized direct comparison heart failure trials. On the other hand, carvedilol and nebivolol showed no superiority over one another, including clinical end points in patients with systolic dysfunction and hypertension $[9,10]$. In AMI patients with an LV ejection fraction $<0.45$, carvedilol and metoprolol showed similar long-term clinical end points, but the safety profile and quality of life were better with carvedilol [7]. In addition, carvedilol and metoprolol exhibited similar short-term clinical end points, reperfusion arrhythmias, cardiac and neurohumoral markers and LV ejection fraction in patients undergoing primary PCI [16]. The long-term clinical end point rates of AMI patients with a mean ejection fraction of 0.55 were also not statistically different between the carvedilol and atenolol groups [17]. The present study is in line with previous studies that show similar event rates with nebivolol and carvedilol. Carvedilol has antioxidant, anti-inflammatory, antiarrhythmic, vasodilatory and $\beta_{1}, \beta_{2}$ and $\alpha_{1}$-receptor blocker properties $[11,18,19]$. Its $\beta_{2}$ receptor blocker and antioxidant effects are critical as $\beta_{2}$ receptors [6] and oxidative stress [27] are increased in heart failure. Previous experimental and clinical heart failure studies indicated the superiority of carvedilol over other BBs and its effectiveness in heart failure with preserved LV ejection fraction [28]. However, direct comparison studies in the AMI settings and a new meta-analysis in the heart failure set- tings showed that the benefits of BBs are mainly due to a class effect with no superiority of any BB over the others [29]. Moreover, beneficial effects of BBs could mainly be related to the degree of the heart rate reduction achieved, which is more successfully achieved with the use of $\beta_{1}$ selective BBs such as nebivolol or metoprolol [16]. In addition, $\beta_{1}$-receptor stimulation increases myocyte apoptosis, but $\beta_{2}$-receptor stimulation has an opposite effect on apoptosis [30]. All of these mechanisms could explain why carvedilol was not superior to nebivolol or metoprolol in AMI patients. In addition, both the COMET and CAPRICORN studies, which showed a superiority of carvedilol over metoprolol or placebo, included patients with clinical heart failure, which was not a required inclusion criterion in the present study or in other studies including AMI patients [7, 16, 17]. We speculate that carvedilol may not be as beneficial in AMI patients as it is in those with chronic heart failure. The limitation of this study was a highly selected cohort group without any controls due to ethical considerations.

\section{Conclusion}

The nebivolol group experienced 12-month cardiovascular events at a lower rate than the metoprolol succinate group. The difference in event rates was mainly due to greater rehospitalization in the metoprolol group. However, event rates were similar between the carvedilol and the metoprolol groups and between the nebivolol and the carvedilol groups. Withdrawal rates were statistically similar among the three groups. If confirmed by a larger study, this would be a clinically important finding.

\section{References}

1 The MERIT-HF Study Group: Effect of metoprolol CR/XL in chronic heart failure: Metoprolol CR/XL Randomised Intervention Trial in Congestive Heart Failure (MERIT-HF). Lancet 1999;353:2001-2007.

-2 Poole-Wilson PA, Swedberg K, Cleland JG, et al: Comparison of carvedilol and metoprolol on clinical outcomes in patients with chronic heart failure in the Carvedilol or Metoprolol European Trial (COMET): randomised controlled trial. Lancet 2003;362:7-13.

>3 The SENIORS Investigators: Randomized trial to determine the effect of nebivolol on mortality and cardiovascular hospital admission in elderly patients with heart failure. Eur Heart J 2005;26:215-225.
4 The CAPRICORN Investigators: Effect of carvedilol on outcome after myocardial infarction in patients with left-ventricular dysfunction: the CAPRICORN randomized trial. Lancet 2001;357:1385-1390.

5 DiNicolantonio JJ, Fares H, Niazi AK, et al: $\beta$-Blockers in hypertension, diabetes, heart failure and acute myocardial infarction: a review of the literature. Open Heart 2015; 2:e000230.

6 Ripley TL, Saseen JJ: $\beta$-Blockers: a review of their pharmacological and physiological diversity in hypertension. Ann Pharmacother 2014:48;723-733.
7 Mrdovic IB, Savic LZ, Perunicic JP, et al: Randomized active controlled study comparing effects of treatment with carvedilol versus metoprolol in patients with left ventricular dysfunction after acute myocardial infarction. Am Heart J 2007;154:116-122.

>8 Triposkiadis F, Giamouzis G, Kelepeshis G, et al: Acute hemodynamic effects of moderate doses of nebivolol versus metoprolol in patients with systolic heart failure. Int J Clin Pharmacol Ther 2007;45:71-77.

9 Marazzi G, Volterrani M, Caminiti G, et al: Comparative long term effects of nebivolol and carvedilol in hypertensive heart failure patients. J Card Fail 2011;17:703-709. 
10 Lombardo RM, Reina C, Abrignani MG, et al: Effects of nebivolol versus carvedilol on left ventricular function in patients with chronic heart failure and reduced left ventricular systolic function. Am J Cardiovasc Drugs 2006; 6:259-263.

-11 DiNicolantonio JJ, Lavie CJ, Fares H, et al: Meta-analysis of carvedilol versus beta 1 selective beta-blockers (atenolol, bisoprolol, metoprolol, and nebivolol). Am J Cardiol 2013;111:765-769.

12 Thygesen K, Alpert JS, White HD, et al: Universal definition of myocardial infarction. Circulation 2007;116:2634-2653.

13 Grossgasteiger M, Hien MD, Graser B, et al: Image quality influences the assessment of left ventricular function: an intraoperative comparison of five 2-dimensional echocardiographic methods with real-time 3-dimensional echocardiography as a reference. J Ultrasound Med 2014;33:297-306.

14 Steg PG, James SK, Atar D, et al: ESC guidelines for the management of acute myocardial infarction in patients presenting with ST-segment elevation. Eur Heart J 2012;33:25692619.

15 Masoudi FA, Bonow RO, Brindis RG, et al: ACC/AHA 2008 statement on performance measurement and reperfusion therapy: a report of the ACC/AHA Task Force on Performance Measures (work group to address the challenges of performance measurement and reperfusion therapy). J Am Coll Cardiol 2008; 52:2100-2112.

-16 Tölg R, Witt M, Schwarz B, et al: Comparison of carvedilol and metoprolol in patients with acute myocardial infarction undergoing pri- mary coronary intervention - the PASSAT study. Clin Res Cardiol 2006;95:31-41.

17 Jonsson G, Abdelnoor M, Muller C, et al: A comparison of the two beta-blockers carvedilol and atenolol on left ventricular ejection fraction and clinical end-points after myocardial infarction: a single-centre, randomized study of 232 patients. Cardiology 2005; 103:148-155.

18 McMurray J, Køber L, Robertson M, et al: Antiarrhythmic effect of carvedilol after acute myocardialinfarction: results of the Carvedilol Post-Infarct Survival Control in Left Ventricular Dysfunction (CAPRICORN) trial. J Am Coll Cardiol 2005;45:525-530.

19 Torp-Pedersen C, Poole-Wilson PA, Swedberg $\mathrm{K}$, et al: Effects of metoprolol and carvedilol on cause specific mortality and morbidity in patients with chronic heart failure - COMET. Am Heart J 2005;149:370376.

20 Sorrentino SA, Doerries C, Manes C, et al: Nebivolol exerts beneficial effects on endothelial function, early endothelial progenitor cells, myocardial neovascularization, and left ventricular dysfunction early after myocardial infarction beyond conventional $\beta_{1}$-blockade. J Am Coll Cardiol 2011;57:601-611.

21 Rousseau MF, Chapelle F, van Eyll C, et al: Medium-term effects of beta-blockade on left ventricular mechanics: a double-blind, placebo-controlled comparison of nebivolol and atenolol in patients with ischemic left ventricular dysfunction. J Card Fail 1996;2:15-23.

22 Dogan A, Karabacak M, Tayyar S, et al: Comparison of the effects of carvedilol and nebivolol on diastolic functions of the left ventricle in patients with non-ischemic heart failure. Cardiol J 2014;21:76-82.

23 Contini M, Apostolo A, Cattadori G: Multiparametric comparison of carvedilol, vs. nebivolol, vs. bisoprolol in moderate heart failure: the CARNEBI trial. Int J Cardiol 2013; 168:2134-2140.

24 Weiss R: Nebivolol: a novel beta-blocker with nitric oxide-induced vasodilatation. Vasc Health Risk Manag 2006;2:303-308.

25 Wei S, Chow LTC, Sanderson JE: Effect of carvedilol in comparison with metoprolol on myocardial collagen postinfarction. J Am Coll Cardiol 2000;36:276-281.

26 Packer M, Antonopoulos GV, Berlin JA, et al: Comparative effects of carvedilol and metoprolol on left ventricular ejection fraction in heart failure: results of a meta-analysis. Am Heart J 2001;141:899-907.

27 Karabacak M, Dogan A, Tayyar S, et al: Oxidative stress status increase in patients with nonischemic heart failure. Med Princ Pract 2014;23:532-537.

28 Yamamoto K, Origasa H, Suzuki Y, et al: Relation of risk factors with response to carvedilol in heart failure with preserved ejection fraction - a report from the Japanese Diastolic Heart Failure Study (J-DHF). J Cardiol 2013; 63:424-443.

29 Chatterjee S, Biondi-Zoccai G, Abbate A: Benefits of $\beta$ blockers in patients with heart failure and reduced ejection fraction: network meta-analysis. BMJ 2013;346:f55.

30 Communal C, Singh K, Sawyer DB, et al: Opposing effects of 1 - and 2-adrenergic receptors on cardiac myocyte apoptosis. Circulation 1999;100:2210-2222. 